\title{
Odres nuevos para viejos brebajes '
}

\author{
J. FRANCISCO ÁLVAREZ \\ UNED
}

En el análisis de las relaciones entre la economía (política) y la filosofia moral resulta casi obvia la conveniencia de remontarse a la obra de Adam Smith, ya sea a su teoría de los sentimicntos morales o a la investigación sobre la naturaleza y causas de la riqueza de las naciones. Quizás haya quienes encuentren más razonable elegir como punto de partida la Politica de Aristóteles, o bien, dependiendo de la propia tradición cultural, elegir de la India clásica el Arthasastra de Kautilya. De todas estas opciones cabe decir, en estos tiempos de clonación, que lo más eficaz para tener un futuro prometedor es elẹgir bien a los propios padres, como irónicamente ha recordado J. Hintikka en referencia a la historia de la lógica. En este número especial de Isegoría aparecen varios artículos que recuerdan temáticamente problemas del pasado, pero bien sabemos que lo que no es tradición es plagio.

Cuando accpté la propuesta del consejo editorial de Isegoría, que continuando con su plan periódico de números monográficos ${ }^{2}$ me ofrecía coordinar este número sobre Etica y economía politica, me planteé solicitar y reunir un conjunto de trabajos que mostrasen la diversidad de temas que vuelven a conectar hoy la práctica de la ciencia económica con las reflexiones éticas e incluso señalar cómo algunos problemas tradicionales de la ética están siendo abordados con nuevos instrumentos metodológicos y formales de la ciencia económica contemporánea. En todo caso es importante observar cómo la doble dirección de las influencias mutuas no ha dejado nunca de estar presente desde la época de cualquiera de los comunes antecesores. Algunos de los textos que aquí aparecen, todos ellos originales para este número, nos incitan y nos facilitan ese recuerdo, otros sin hacerlo explícito lo muestran claramente en la práctica para el lector bien avisado.

Vínculos temáticos estrechos con la tradición aparecen en el trabajo de Elliot Sober, destacado filósofo de la biología, quien nos presenta un detallado

'A la par que se procede a presentar los artículos de este número, se avanzan algunas tesis que utilizan y reelaboran algunos de mis trabajos previos. En particular uno que tuvo una primera presentación pública como «Nuevos vínculos entre ética y economía», y fue mi contribución al volumen colectivo Filosofia moral, educación e historia (México, 1996), editado por León Olivé y Luis Villoro como homenaje al profesor Fernando Salmerón.

"Sc cditó en noviembre de 1992 Feminismo y ética, y en octubre de 1995 La filosofía de la ciencia como fillosofia práctica, números respectivamente 6 y 12 de la revista. 
análisis, claro y sencillo, sobre la posibilidad de defender racionalmente el pluralismo de los motivos o, al menos, no avalar sin más el egoísmo psicológico como elemento de sentido común a partir del cual montar las diversas teorías económicas. Aquí encontramos ya un elemento básico de por dónde hay que proceder hoy a la reconstrucción y crítica de la actual ciencia económica. Cierta vuelta a la inspiración básica de Smith para construir una crítica teórica y empírica de los fundamentos mismos, y de los supuestos metodológicos, de la ciencia económica. De ahí la relevancia del trabajo de Sober, que supone un avance teórico importante para robustecer la crítica sistemática realizada desde diversas propuestas éticas a la teoría económica estándar. Fuertes conexiones con esa propuesta de Sober aparecen, en parte como consecuencia inesperada para el editor, en la colaboración de Elizabeth Anderson y en la de Félix Ovejero.

Aunque temáticamente hay relaciones con el pasado, sin embargo las herramientas utilizadas en la mayor parte de los artículos se corresponden con buena parte del instrumental desarrollado por la ciencia económica en el último siglo. Un caso ejemplar se muestra en el trabajo de Juan Urrutia. El análisis de las instituciones y, en particular, la precariedad de alguna de ellas (caso del Banco Central) lo realiza el autor mediante un sutil uso de la teoría de juegos, señalando sus limitaciones, incorporando las revisiones más recientes de esa teoría y revisando las nociones de racionalidad utilizadas.

Muy pertinentes resultan las reflexiones metodológicas que nos propone Toni Domèncch. La preocupación por temas metodológicos ha sido constante en la ciencia económica y buena parte de esos debates han contribuido a precisar el ámbito y la cientificidad de los estudios sociales. En relación con la importación de procedimientos procedentes de la economía nos encontramos, en tanto que filósofos de la ciencia e incluso como filósofos morales, ante una situación similar a la que se produjo debido al boom de la sociología de la ciencia. Parece conveniente incorporar los resultados de la ciencia económica, a la par que resistimos al afán de expansión imperialista de sus métodos. Quizá valga la pena importar algunas formas de razonamiento, cierta manera de ver los problemas, pero con consciencia clara de las dificultades y limitaciones que esos mismos métodos han encontrado en la propia ciencia económica.

El trabajo de John Roemer que publicamos supone también una contribución destacada de un nuevo estilo de hacer economía política, en que se abordan los problemas de la igualdad y la libertad, las supuestas contradicciones de ese binomio y el estudio concreto de modelos que unen la precisión analítica con la preocupación política por los otros. Se trata de un trabajo representativo de los últimos avatares de lo que se dio en llamar marxismo analítico y ha pasado hoy a ser una línea de reficxión interesante sobre filosofía moral realizada con herramientas procedentes en su mayor parte del análisis económico.

Entre los trabajos técnicos en economía podríamos destacar dos líneas de pensamiento que son muestra de demandas que la economía hace a otros 
campos del conocimiento y que reflejan que el problema no se reduce al del imperialismo de los métodos económicos sobre la ética. Me refiero a que buena parte de lo que se relaciona con el estudio de la incertidumbre, con los problemas de la economía institucional y con la teoría de la elección pública está demandando la contribución de otros campos, y muy en particular precisa de la reflexión ética. Podríamos hacer nuestras las palabras de John Hey: «A juzgar por el material reseñado en este capítulo, bien pudiera ocurrir que los economistas necesitasen ayuda de otras disciplinas para modelar apropiadamente la incertidumbre en la cconomías (John D. Hey, Companion to Contemporary Economic Thought, Routledge, 1991, p. 273); disciplinas que bicn pudieran ser la filosofía moral o la filosofía política.

La vía seguida por ciertos lamentos filosóficos, que todo lo interpretan en términos de la crisis de la filosofia, la muerte de las idcologías, cl fin de la historia, o cosas por el estilo, no me parece una senda interesante de conocimiento; por el contrario, tengo por más prometedor el intento de deslindar algunos aspectos en los que la filosofía ha tenido un relativo éxito para estimar nuestro trabajo actual. Hemos querido dejar reflejadas en las reseñas de libros algunas contribuciones recientes a estos problemas de la relación entre ética y economía, sobre todo cuando han producido resultados fecundos debidos a dicha interrelación. Aunque podrían entrar otros nombres, hemos optado por presentar muestras de un grupo de autores que están trabajando en esa frontera de la articulación entre ética y economía, sin que falten en nuestra lengua, y a ambos lados del Atlántico, quienes en esa línea tratan de ir bastante más allá de sugerir propuestas deontológicas para empresarios bien intencionados. Quizá sea el momento de ponerse a trabajar en una tarea como la propuesta por Partha Dasgupta en su importantísimo libro Investigación sobre el bienestar y la miseria, donde se nos recuerda que disponemos ya de una amplísima cantidad de datos relativos a las condiciones de vida de los más pobres entre los pobres, así como abundante material acumulado sobre aspectos relevantes de los diversos mecanismos para la asignación de recursos que se han aplicado durante amplios períodos en diversos países. Es momento de poner esos datos en contacto con la reflexión sobre aquelios aspectos de la condición humana que los filósofos modernos han mostrado que tienen carácter o validez universal.

Como el mismo Dasgupta señala, en ciencias sociales y en filosofia se han adoptado dos puntos de vista bien diferentes sobre las personas. Una inadecuada percepción del punto de vista desde el que se hace el análisis ha producido confusiones, aunque de muchas de ellas pueda decirse que han sido confusiones razonables. Por ejemplo, con frecuencia a la hora de hablar de las relaciones entre ética y economía se ha adoptado una perspectiva sobre el ser humano que parece admitir la simple generalización del uso de los instrumentos formales de la ciencia económica para abordar las reflexiones éticas. Se ha tratado de cambiar el modelo de persona y adoptar el que ha sido dominante en el campo 
de la economía. Nos interesa destacar la importancia que puede tener el camino inverso, a saber, abordar temas de primera importancia en la economía mediante el uso de un modelo de persona cuya adopción es más usual en el campo de la ética, sin que por ello se rechace la importancia que para las cuestiones éticas pueda tener la adopción del punto de vista tradicional de la economía. Partha Dasgupta distingue bien esos dos aspectos: «Uno nos considera como seres capaces de deliberar y de tener la capacidad potencial de hacer cosas, por tanto, se centra en el sujeto agente, la elección, la independencia, la autodeterminación y los aspectos de nuestro yo que contribuyen a que modele proyectos y persiga objetivos. La otra perspectiva nos ve como asentamiento de utilidad y de satisfacción, como lugar de posibles estados mentales descritos por la amplitud en la que se satisfacen los deseos mediante las diversas actividades que se realizan y las relaciones que se mantienen. Una de las perspectivas nos ve haciendo cosas, la otra nos percibe como colocados en una determinada situación.» La primera orientación nos conduce al lenguaje de la libertad y de los derechos, la segunda directamente al lenguaje del bienestar. En relación con este tema resultan enormemente significativas las aportaciones de Elizabeth Anderson: la que aquí publicamos es muestra de su finura intelectual a la par que exhibe con todo detalle la complejidad en la que se han situado este tipo de discusiones. La noción de «autonomía de nosotros o autonomía de colectivos» permite cierta salida a las aparentes paradojas entre la soberanía del consumidor y la soberanía del ciudadano que, junto a las propuestas sobre el pluralismo motivacional de Sober, apoyado en la biología evolucionista, constituyen una crítica y una contribución de primer orden a los fundamentos mismos del análisis económico.

Ciertos temas aparentemente clausurados, como ocurre con la relación existente entre el individualismo metodológico y otros presupuestos más generales sobre la naturaleza humana, demandan, sin embargo, ulterior reflexión a partir de las críticas relaciones entre ética y economía. Son problemas que tienen múltiples antecedentes históricos y que aparecen en formas contemporáneas algo diferentes, aunque a veces éstas ni siquiera sean muy diversas de las que los recubrían tradicionalmente. La discusión ahora me parece que se desarrolla siguiendo el camino de la ética a la economía. Buena parte de estos problemas aparecen en la discusión llevada a cabo en este número entre Julia Barragán y Pedro Francés, donde se evalúan con suma atención los éxitos y posibles deméritos de la tradición utilitarista, desde el mismo interior de la tradición (Barragán) y un poco más exteriormente (Francés).

Al analizar los condicionantes de nuestra acción aparecen un conjunto de problemas que genéricamente podemos clasificar de asuntos metaéticos. Entre algunas de esas propuestas metaéticas vale la pena destacar las tesis de Amartya K. Sen, que, aunque no se presenten aquí en toda su extensión y complejidad, aparecen reflejadas en diversas partes de casi todos los artículos (de ahí la pertinencia de reseñar algunas de sus últimas contribuciones). Amar- 
tya Sen ha insistido sobre el carácter de «filtro informativo" que adoptan los principios éticos, ha señalado cómo se puede aplicar esa perspectiva para analizar las consideraciones de justicia y, al mismo tiempo, ha puesto el acento especialmente sobre la noción de «capacidades potenciales», lo que viene a ser tanto como asignarle un importante papel a las libertades concretas y a la propia responsabilidad del sujeto como agente ${ }^{3}$.

Para nuestra discusión presente puede que no sean del todo pertinentes, al menos pueden ser dispensables, algunas cuestiones formales que acompañan a las propuestas de los economistas. Por ejemplo, la posibilidad de abordar en términos de orden parcial aspectos muy discutidos sobre las diversas formas de consideración del propio bienestar e incluso el complejo asunto de la comparación interpersonal de utilidades. Baste señalar que la noción de retículo, y las álgebras booleanas aplicadas a una ordenación parcial del conjunto de preferencias, puede ser un modclo adecuado para analizar algunas propiedades de un espacio de elección evaluado moralmente y para organizar lo que podríamos llamar una topología moral ${ }^{4}$. Pero se puede reflexionar más sobre estos temas a partir de la sintética y esclarecedora contribución de Salvador Barberá, quien al formalizar la noción de incertidumbre, entendida como un tipo de ignorancia cualitativamente diferente del ricsgo, apunta dentro de unos compromisos muy austeros (expresados en unos axiomas muy poco restrictivos) al valor de la libertad de elección y muestra la importancia de atender al conjunto de capacidades potenciales en lugar de a la simple elección.

El grueso de la posición de Sen se puede articular en torno a su propuesta metaética de considerar a los principios morales desde el punto de vista de la información. Se trata de una aparente trivialidad, una llamada de atención sobre a qué aspectos atendemos y a cuáles no:

«El fundamento o apoyo informativo de un juicio identifica la información de la que depende directamente el juicio y - no menos importante- afirma que la verdad o falsedad de cualquier otro tipo de información no puede influir directamente en la corrección de tal juicio. La base informativa de nuestras consideraciones o juicios de justicia determina así el territorio fáctico sobre el cual son directamente aplicables esas consideraciones de justicia (las implicaciones sobre otras materias deben ser derivadas)» (A. Sen, «Justice: Means versus Freedoms», p. 111).

${ }^{3}$ Para esta presentación, además de los principales trabajos de Sen sobre estos temas elaborados en los últimos veinte años, tienen interés algunos de sus trabajos de divulgación, como el artículo del New York Review of Books correspondientes al 14 de junio de 1990, «Individual Freedom as a social commitment». El material bâsico se encuentra en su artículo «Justice: Means versus Freedoms», Philosophy and Public Affairs, primavera 1990, vol. 19, núm. 2, dedicado básicamente a la continuación de su debate con J. Rawls, y en «Minimal Liberty», Economica, núm. 59, pp. 139-159, mayo 1992, y, por supuesto, en su libro Inequality Reexamined, Oxford, 1993.

"Carlos Thiebaut habla de «tropologia moral» para indicar el espacio de lo estético que puede incidir en el tema de la ética. Aquí, sin embargo, se trata de una complejidad formal del individuo que puede llamarse su estructura topológica y que reflejaría las posiciones duales, imparciales y subjetivas que adopta. 
La propuesta de Sen, aunque él mismo se considera deudor de la obra de Rawls en ciertos elementos estructurales, se diferencia por señalar que si bien las comparaciones interpersonales constituyen elementos decisivos en la fundamentación informativa de la justicia, sin embargo, tal fundamentación no puede obtenerse a partir de comparaciones efectuadas sobre los medios para la libertad (sean recursos, ingresos o bienes primarios). El fondo de la propuesta de Sen consiste en defender que una teoría de la justicia como equidad debería incorporar, de manera directa y básica, las libertades concretas y efectivas que pueden ser disfrutadas por personas diferentes, personas con objetivos posiblemente diversos, razón por la que es importante considerar el grado preciso de las libertades de que disponen para poder proponerse vivir vidas diferentes. Unas vidas diferenciadas que, además, cada cual puede tener diferentes razones para valorar.

Me parece, por tanto, que tiene importancia y que no es algo marginal observar cómo Sen critica cierto «fetichismo de la mercancía», que está presente en la propuesta de igualdad que se apoya o que está sujeta a una visión derivada de los bienes primarios cuya intercambiabilidad equipara el valor de los bienes para todos. La materialidad diferenciada de la transformación de esos bienes en la obtención concreta de libertad hace importante el análisis de las libertades obtenidas por cada uno, un análisis que no debe quedar ceñido a los medios para posibles libertades. Este problema es parecido al de la conversión de trabajo heterogéneo en trabajo abstracto, una dificultad bien conocida en el campo de la economía marxista y que se repite aquí ante una dificultad similar a la que aparece en la formación de cualquier índice a partir de dotaciones heterogéneas. La tendencia a calcular los valores en términos de una única medida, ya sea la cantidad de trabajo abstracto o la búsqueda de un único referente en términos de utilidad, se plantea casi siempre con los mismos esquemas formales e ideológicos: la posibilidad de un único rasero. La cuestión importante para la ética y, finalmente, para la economía, es más bien la del pluralismo y la desigualdad, ahora considerados como un resultado tenaz y no como un resultado temporal, a falta de disponer, por el momento, de otro criterio general mejor que permitiese realizar la comparación. Me parece que lo importante es encontrar procedimientos de comparación interpersonal, pero no tanto de las utilidades comunes, sino de la diferencia, de la pluralidad y la peculiaridad. Es una propuesta que se alcjaría de cualquier ascetismo pretendidamente generalizable. Una propuesta que se aleja de los modelos únicos que, con harta frecuencia, reiteran la falacia de composición al proponer generalizar lo que es posible para cualquiera sin percibir que quizá no sea posible simultáneamente para todos.

Propongo, pues, analizar las restricciones informativas en un sentido bien diferente a quicnes sugieren que es la misma sociedad de mercado la que pone dificultades al flujo de cicrto tipo de información referente a los individuos. En este aspecto me apoyo en trabajos de Elizabeth Anderson, que ella misma 
desarrolla ahora más en su contribución a este número de Isegoría. Cicrtos ascetas que a la vez son defensores a ultranza del mercado proponen una tarea imposible para seres humanos normales; trascender la situación de limitaciones informativas del mercado por la vía de la profundización racional individual, lo que no es sino una forma extravagante de hiperracionalismo por su imposible generalización, y creo que reflejan la incidencia, aunque no intencional, sobre la economía de nociones heredadas de la ética. Con esa actitud se impide el registro de algunos otros elcmentos informativos, elementos que se corresponden con el campo de «autonomía de la ideología» y con las consideraciones autónomas de lo moral. Es posible que estos rasgos puedan también tener su correspondiente indicación contable, a veces incluso a partir de los mismos indicadores. Puede encontrarse cierta similitud entre nuestra propuesta y la que ha realizado J. Muguerza al analizar un momento de la abstracta reconstrucción histórica de la palabra «bueno»: «Habria que contar con la intromisión de la tradición judeocristiana, para la que algo es bueno si así lo quiere Dios, lo que plantea el arduo problema de si es bueno porque lo quicre Dios o más bien Dios lo quiere porque es bueno" (Desde la perplejidad, p. 182), lo mismo que con el matiz que $A$. Sen trata de destacar al señalar que la satisfacción de los deseos indica una valoración por nuestra parte: «lo descamos porque lo valoramos, o como lo consideramos bueno lo deseamos» (A. Sen).

Surge asî otra cuestión importante: no basta con controlar nuestras aspiraciones y ser un asceta (recuérdese la crílica de Marx a la concepción del trabajo como sacrificio: flagelarse no es trabajo) para que cuente como satisfacción de nuestras necesidades la pura y simple supresión de ellas. La contabilidad del consumista suntuario y la del asceta serían situaciones similares: en ninguno de ambos casos parece fácil aceptar el proceso aditivo para contabilizarlos como logro social ni se puede pretender la imposición y generalización del modelo; aparecerían así las diversas formas del autoritarismo (sea ecologista, comunitarista o ilustrado); en definitiva, la planificación racional apareccría como criterio del nuevo dictador.

La base informativa sirve para reflexionar sobre los elementos que las diversas teorías utilizan para apoyar sus juicios ćticos sobre las personas. Al analizar diferentes propuestas sobre la justicia, Amartya Sen se fija en dos tipos básicos de información relevante: a) los rasgos personales sobre los que centramos la atención - rasgos focales-, y b) las características de los procedimientos de combinación. En suma, creo que no se trata sino de presentar con claridad los elementos de un determinado conjunto y la ley de composición que permite dotar de estructura a ese ámbito. La propuesta concreta de Sen consiste en proceder a la evaluación de la justicia sobre la base de las capacidades, es decir, centrarse sobre aquellos aspectos personales que se refieren a las libertades concretas que efectivamente disfrutan los individuos, para poder elegir entre maneras de vivir que se pueden considerar como atractivas o valiosas. 
«Esa libertad concreta, real, está representada por la "capacidad potencial" o "aptitud" de las personas para lograr varias combinaciones alternativas de funcionamientos, es decir, de sus formas de ser y de actuar. Por ello se trata precisamente de distinguir entre medios para la libertad y libertad y, también, entre libertad y los logros concretos.»

La cuestión, insistirá Sen en discusión con Rawls, es que debemos distinguir entre libertad (de la cual la «capacidad potencial» es una representación) y el logro o éxito concreto. La «capacidad» refleja la libertad de una persona para elegir entre vidas alternativas, es decir, entre determinadas combinaciones de «funcionamientos». Al atender a la capacidad potencial, y debido a que hay condiciones diferentes entre los individuos, es preciso considerar la transformación en logros concretos que cada uno realiza de los bienes primarios. Mientras que los bienes primarios nos hablan sólo de los medios para la libertad, sin atender a la variación interpersonal entre esos medios y las libertades concretas conseguidas, la capacidad representa la libertad. Utilizando términos de Sen:

«Somos diversos, pero lo somos de maneras diferentes. Un cierto tipo de variación se relaciona con las diferencias que hay entre nuestros fines y objetivos. Las implicaciones éticas y políticas de esta diversidad las entendemos ahora mejor que antes como resultado de los potentes trabajos de Rawls sobre la justicia como equidad. Pero hay otra diversidad importante - las variaciones en nuestra aptitud para convertir recursos en libertades concretas-. Variaciones que hacen referencia al sexo, a la edad, a la dotación genética y a muchos otros rasgos que nos dan potencia desigual para construir nuestra libertad en nuestras vidas, aunque tengamos la misma dotación de bienes primarios.»

En esta misma dirección me parece posible defender cierto refinamiento de la noción de individualismo metodológico. Una posibilidad que surge ahora desde la consideración del individualismo ético. La valoración, en último término, de la responsabilidad individual fortalece nuestra concepción del individualismo metodológico; no se trata de que uno de los términos implique al otro, ni mucho menos. Pero, así como se sostiene a veces cierto individualismo metodológico bastante radical considerando, por ejemplo, que «el comportamiento egoísta es simplemente una consideración metódica», es importante darse cuenta de que con ello no se dice sólo que el individuo es el sujeto de deseos, creencias y decisiones, sino que se le añade cierta caracterización que se corresponde de forma no neutral con unas nociones éticas bien específicas. En particular se incorporan nociones heredadas del utilitarismo moral, que han influido de manera especial sobre la ciencia económica y desde ella vuelven al análisis moral. No sólo se ha producido la extensión imperial de la ciencia económica hacia la ética, sino que se expande el individualismo posesivo como acompañante suyo. Se plantea, en esas propuestas, la exigencia de que el individuo en tanto que elemento moral asuma los valores conformados de manera dominante por alguna escuela de pensamiento económico o por 
alguna norma social. En la contribución de Sober a este número se fundamenta con todo detalle la posibilidad de esta crítica al pensamiento económico estándar. Incluso puede decirse que Sober realiza un desplazamiento de la tópica discusión sobre el individualismo metodológico y ofrece material para establecer un puente entre diversas formas del individualismo y el holismo sin hacer concesiones a ninguna de las partes.

Me parece que es posible un refinamiento del individualismo metodológico que recoja su inspiración de otra dirección del pensamiento ético, una orientación, atenta a los diferentes rasgos individuales a la hora de realizar una apreciación ética, que se preocupe por la autonomía del individuo como agente moral en un contexto de interdependencia. A partir de ese individualismo, ahora ya claramente ético, con alta complejidad formal, complejidad que es sin duda la manera peculiar de presentarse el «contenido", la vieja discusión sobre el formalismo moral o el sustantivismo moral puede ser planteada desde otra óptica. «O se legisla para todo hombre o sencillamente no hay ética posible (...). [Pero] puesto que el uso moral de la palabra "bueno" nos implica del modo inexcusable que hemos visto, o aceptamos autodeterminarnos - de suerte que cada hombre sea un legislador - o toda legislación ética se tornaría, como antes, imposible» (Javier Muguerza, Desde la perplejidad, pp. 183-185). Que los límites de nuestra acción racional señalan límites a la forma que puede adoptar nuestra propia valoración moral es, desde luego, un elemento que ha sido suficientemente señalado, en particular debido a los productos esencialmente laterales de nuestra acción; la cuestión reside en que, si nuestra evaluación moral pretende sustentarse en alguna noción de utilidad como satisfacción de nuestros deseos, la ausencia de un curso óptimo de acción no sólo rompe la racionalidad de la acción, sino que quiebra nuestra misma caracterización ética. Pero no podemos aceptar la simpleza, defendida por cierto tipo de decisionismo moral, de que cuando no se puede discutir racionalmente sobre algo es ahí donde aparece el espacio de la ética, puesto que más bien se trata de lo contrario. Cuando no hay condiciones para proceder a la discusión racional no es fácil ver dónde se sitúa el comportamiento ético, ya que se trata de una situación pre-ética; la ética es necesaria para orientarnos en momentos en los que no basta con los elementos de racionalidad mínima supuestos en el modelo de acción racional habitual en la teoría de la elección. Este tema, aunque con otras claves, es uno de los desarrollados brillantemente por Félix Ovejero en su contribución al presente número.

Con frecuencia ese papel activo en la búsqueda del acuerdo lo adopta la ética mediante el oficio de filtro informativo que juegan los principios éticos. Filtros informativos que pueden servir para indicar el grado de satisfacción de un objetivo, en lugar de pretender conseguir un inencontrable curso óptimo de acción, y para actuar como mecanismo cuasi causal que determine la elección entre varios cursos posibles racionales de la acción (seguramente la noción de centroide de Harsanyi podría aparecer aquí como relevante). Además, las 
propuestas éticas pueden servir con frecuencia para resolver los problemas que aparecen en la búsqueda de la información y para «justificar» por qué hay cierto tipo de información que no recogemos -recogemos la evidencia necesaria para confirmar nuestras creencias y para avanzar en nuestras aspiraciones.

Posiblemente una reconsideración más sofisticada del individualismo metodológico podrá presentarse a condición de que seamos capaces de refinar nuestra noción de individualismo ético, y esto nos diferencia de quienes piensan que al lenguaje moral se le exige la universalizabilidad. Al eliminar todo lo referente al individuo, la cxpresión universal es una simple consecuencia fáctica de esa eliminación, por lo que nunca podríamos recuperar al individuo concreto. La opción del individualismo ético, al introducir en último término las exigencias del propio individuo, no produce un yo universal o universalizable a partir de contenidos concretos, aunque sí puede definir un conjunto mínimo de exigencias formales. El individuo formal abstracto no tiene por qué ser un individuo plano y romo obligado a considerar una exclusiva relación de preferencia, sino que puede tener una familia de preferencias y entre ellas puede incluir su propia libertad. Es decir, la complejidad de la consideración de los individuos se produce encontrando nuevos estratos, pero también ampliando el campo de lo abordable y asignando otro tipo de funciones de valoración. La aportación del individualismo ético no consecuencialista produce un punto de vista sobre lo social que añade elementos al significado del bien para el individuo en la definición del bien social, y me parece que en buena medida varias de las contribuciones a este número pueden ayudar a cste tipo de reflexión, aunque no esté claro si todos los autores apoyan por igual esta salida. La contribución de Paulette Dieterlen es significativa y muy esclarecedora al respecto.

Además, debemos tener en cucnta que nuestra misma definición como racionales es producida por el hecho moral, y ello a pesar de que: «Para un representante del mejor liberalismo, como Kant, el último sentido de la libertad y la igualdad - esto es, de la exigencia de que los seres humanos sean libres e iguales - radicaba ni más ni menos que en nuestra condición de seres racionales» (Desde la perplejidad, p. 181). Pues bien, en la interpretación que defendemos es posible que la consciencia sobre cómo plantear la propia racionalidad nos haga menos orgullosos sobre nuestra capacidad de alcanzar la bondad. Al mismo tiempo quizá nos permita una caracterización más adecuada de la acción justa; digamos que no sólo amplía las libertades negativas, sino que incorpora las libertades positivas.

En esos límites de la racionalidad aparecen algunas cuestiones que deben precisarse: en particular, las nociones de racionalidad limitada y racionalidad imperfecta - dos conceptos muy diferentes-, la satisfacción de algún principio en lugar de la optimización, el papel de la discusión racional en la conformación de las creencias y los deseos, y el importante asunto de las emociones y las pasiones. Temas que, de nuevo, se ven muy esclarecidos en la ya citada contribución de Félix Ovejero. 
Situados aquí nos reencontramos con el papel que desempeñan los principios ćticos en la recopilación y selección de información relevante, pero, sobre todo, con cómo se aplican para determinar el conjunto de información que se climina. Así como se habla de infradeterminación de la teoría por los hechos, en el sentido de una posible variedad de construcciones teóricas a partir de un conjunto similar de datos empíricos 0 , al menos, a partir de un mismo conjunto de conceptos no teóricos, tendríamos también una infradeterminación del principio moral por parte de nuestros datos. Hay diversos principios que se corresponden con un mismo conjunto de datos, o que pueden cubrir por igual a ese conjunto, pero lo importante es que los principios actúan como filtros y sólo dejan pasar cierto tipo de información; no es que los principios incluyan los mismos datos, sino que actúan seleccionando lo que consideramos pertinente. Incluso, a veces, se solapan principios diferentes, pero en otras ocasiones pueden ser independientes. No basta pensar en ciertos tapones informativos; por ejemplo, suponer que el mercado no suministra ciertos datos sólo significa que nuestros principios éticos no se modelan exclusivamente a partir de los datos que nos ofrece el mercado. En algunos casos el conflicto es aparente, porque diversos principios que parecen oponerse ni siquiera se solapan, es decir, hablan de cosas diferentes. Otras veces hay verdadero conflicto, precisamente cuando se solapan. Hay que incorporar elementos histórico-sociales para que unos principios puedan resultar mejores que otros. En ocasiones tratamos de analizar algunos de los resultados obtenidos, iniciando así una forma especial de discusión racional en ética: cierto tipo sofisticado de consecuencialismo ético. Otras veces, pensar en los límites mismos de la racionalidad nos conduce a una repetición del debate entre el optimista y el pesimista; con frecuencia ésa es la única diferencia, pero puede ser puro hiperracionalismo empeñarse en scguir buscando apoyos racionales. Podemos volver a hablar de infradeterminación, aunque en cste caso sea de infradcterminación de la conducta a partir del principio moral; no existe una determinación univoca de la acción, entre otras cosas porque los elementos que conducen a la acción no forman parte sólo de las creencias del individuo y de sus deseos de cumplir con una de sus creencias morales, sino que dependen del grado en que se recoja la información relevante; la misma aspiración o deseo es lo que orienta la selección de información. Sin duda hay rasgos de pensamiento desiderativo en la conducta moral.

«Hay una distinción efectiva entre los aspectos positivos y negativos de la libertad, pero esos diferentes aspectos pueden estar estrechamente interrelacionados e interconectados. Concentrarse sólo sobre uno de ellos resulta ćticamente incompleto, y además puede ser socialmente destructivo. El compromiso social con la libertad individual tiene que preocuparse tanto de las libertades ncgativas como de las positivas, y de sus interconexiones extensivas» (A. Sen, Individualism as a social commitment).

Nos interesa señalar la importancia de incorporar en la evaluación global de la situación el papel del individuo como sujeto agente, como autor, como 
artífice de sus propias decisiones morales; la consideración del individuo como actor de una acción intencional cuyos resultados no podemos sin más prever, determinista y consecuencialistamente, parece apoyar al principio de responsabilidad moral, con lo cual se hace preciso valorar además de los resultados la posibilidad misma del ejercicio de libertades concretas por parte de los individuos. Para este tipo de consideraciones resultan de nuevo indispensables los trabajos de Amartya Sen - - sin duda uno de los nombres claves en la teoría de la elección social-, quien, como él mismo dice, es un economista de profesión que con frecuencia se toma la libertad de meterse de lleno en los debates éticos.

Sin duda, el juicio moral lo realiza un individuo, pero el tipo de información que ese individuo está en condiciones de utilizar, o que está dispuesto a utilizar, depende en gran medida del tipo de principios morales que adopta; principios que en muchos casos le vienen dados como constricción informativa social. Para este asunto vuelvo a llamar la atención sobre la interesante contribución de Elizabeth Anderson. Aunque metodológicamente lo previo sea el individuo $y$, sin lugar a dudas, lo previo éticamente, sin embargo lo que podemos llamar producción social del conocimiento, y la normatividad producida socialmente, son factores decisivos en la constitución del propio individuo como sujeto moral. Además de los efectos no previstos y perversos desde el punto de vista social que resultan cuando se adopta un principio de acción racional individual, también podemos hablar de efectos perturbadores de la conciencia moral y, sobre todo, del consecuencialismo moral si pretendemos vincularlo siempre con el individualismo ético. Son efectos perturbadores que resultan en buena medida de cierta confianza en nuestra capacidad previa de evaluar los resultados, cuando lo cierto es que estamos ante situaciones esencialmente de incertidumbre, ante las cuales lo que importa es sostener nuestros propios principios de autonomía moral.

La consideración del individuo como agente moral, y la correspondiente valoración de su carácter de sujeto agente de la acción moral, nos lleva a incorporar un tipo de información que valora los derechos del individuo y los ámbitos de su libertad, y a que tal información sea considerada un elemento decisivo para realizar una consideración moral, puesto que no serán sólo los elementos referidos al bienestar y a la utilidad social los que entren en la consideración del hecho moral.

La autonomía del aspecto agente de una persona, que tiene el efecto de hacer inaceptable la adopción del bienestar como fundamento informativo para el razonamiento moral substantivo, lleva asimistno a la consecuencia de que el aspecto del bienestar de una persona debc verse tanto en términos de libertad como de logros efectivos.

Como han señalado Daniel Hausmann y Michael McPherson, editores desde 1985 de la importante revista Economics and Philosophy, en su reciente ensayo «Economics, rationality and ethics», aunque muchos cconomistas ven a su cien- 
cia como la ciencia "positiva» de cicrto tipo de fenómenos sociales, la cconomía está construida en torno a una teoría normativa de la racionalidad. De manera que cualquiera que se preocupe por los problemas metodológicos tiene que enfrentarse con csto.

Adam Smith, Karl Marx o Stuart Mill son autores del siglo xIx que tuvieron en cuenta esa conexión y a quienes deberíamos considerar como filósofos morales y políticos además de economistas.

Insistamos en la conveniencia de atender al doble camino de la relación entre ética y economía. Diversos economistas han hecho esfuerzos para señalar la relevancia que la ética tiene para la economía, tanto para la explicación positiva de las elecciones económicas cuanto para las teorías relativas al bienestar económico. Son conexiones que ponen en primer plano las diferencias metodológicas que tiene la economia con respecto a las ciencias físico-naturales. Como han recordado Hausmann y McPherson, unos podrán preocuparse más de los aspectos que hacen metodológicamente similar a la economía con las ciencias naturales y otros atender a los aspectos éticos de las decisiones económicas. Pero el problema es más radical: se trata de señalar que es difícil mantener separadas las cuestiones metodológicas de la economía de las cuestiones evaluativas relativas a las elecciones individuales, de sus constricciones y ramificaciones. Además, las influencias no son unidireccionales, pues también se ha pensado la ética a partir de ciertos resultados de la ciencia económica. En todo caso, como es un aspecto problemático, lo que puede hacer la economía es señalar la ambigïedad y la imprecisión de ciertas propuestas éticas; no se trata de obtener un resultado formal, un teorema que nos señale la relevancia y la «mejor» opción ética, sino que puede servirnos para precisar mejor algunos de los elementos planteados en teoría ética.

Quedan por estudiar otros aspectos de las relaciones entre la economía, la actividad económica y la ética. Algunos de ellos constituirían otra narrativa, otra historia, que queremos dejar abierta para otra ocasión. Me refiero, en particular, a la necesidad de estudiar con mayor detenimiento la actividad persuasiva del discurso económico y la actividad persuasiva del propio discurso ético, para desde ahí encontrar elementos para una nueva conversación. 